\section{MS5-P3 Structural characterization of}

Helicobacter Pylori pathogenic proteins. The case of $\alpha$-carbonic anhydrase

Maria Elena M.E. Compostella ${ }^{1}$, Giuseppe Zanotti ${ }^{1}$

1. Department of Biomedical Sciences, University of Padova

email: elena.compostella@gmail.com

Helicobacter Pylori is a gram-negative, flagellated, spiral-shaped bacterium, able to colonize the human stomach, a very peculiar niche. It affects more than half of world's population. Its infection can lead to severe gastroduodenal diseases, like chronic gastritis, gastric and duodenal ulcer, gastric adenocarcinoma and mucosa-associated lymphoma. Therefore, H. Pylori contagion is a worldwide problem. Several bacterial species are able to transit the human stomach, but only one, H. Pylori, survives intragastric acidity long enough to establish a chronic colonization. There are several mechanisms involved in the acidic acclimation; particularly important in this aspect are the enzymes urease and carbonic anhydrase. Two different carbonic anhydrases (CA) are coded by Helicobacter Pylori: the alpha-CA, with periplasmic localization, and the beta-CA, with cytoplasmic localization. Carbonic anhydrases are metalloenzymes that catalyze the hydration of carbon dioxide to bicarbonate, crucial for the neutralization of the $\mathrm{pH}$ and the consequent survival of bacterium in the acidic environment of the stomach. The active site of the alpha-CA consists of a $\mathrm{Zn}^{2+}$ ion coordinated by three histidine residues. This enzyme is inhibited by many sulfonamide/sulfamate compounds and the growth of the pathogen can be blocked; thus alpha-CA represent a promising drug target for the treatment of patients infected by drug-resistant strains. $H$. Pylori alpha-CA was cloned as fusion protein with 6-His-tag in the pETit C-His vector, without the signal peptide at the $\mathrm{N}$-terminus. The plasmid was transformed in the recombinant Escherichia Coli strain BL21(D3). The protein was purified by IMAC chromatography and gel-filtration. The His-tag was removed by incubation with TEV protease. Crystals were grown by vapor diffusion sitting drop technique. The diffraction data were collected at $1.5 \AA$ resolution and the three-dimensional structure was solved by molecular replacement.

Keywords: Helicobacter Pylori, carbonic anhydrase, metalloenzyme, acidic acclimation
MS5-P4 NowGFP: a green fluorescent protein with an anionic tryptophan-based chromophore

Sergei Pletnev ${ }^{1,2}$, Nadya V. Pletneva ${ }^{3}$, Karen S. Sarkisyan ${ }^{3}$, Alexander S. Mishin ${ }^{3}$, Konstantin A. Lukyanov ${ }^{3}$, Ekaterina A. Goryacheva ${ }^{3}$, Zbigniew Dauter ${ }^{1}$, Vladimir Z. Pletnev ${ }^{3}$

1. Macromolecular Crystallography Laboratory, National Cancer Institute, Argonne IL, USA

2. Basic Research Program, Leidos Biomedical Research, Inc., Argonne IL, USA

3. Shemyakin-Ovchinnikov Institute of Bioorganic Chemistry, Russian Academy of Sciences, Moscow, Russia

email: pletnevs@mail.nih.gov

A green-emitting fluorescent variant NowGFP with the tryptophan-based chromophore (Thr65-Trp66-Gly67) was recently developed from cyan mCerulean by introducing 18 point mutations. NowGFP is characterized by bright green fluorescence at physiological and higher $\mathrm{pH}$ and by weak cyan fluorescence at low $\mathrm{pH}$. Illumination with blue light induces irreversible photoconversion of NowGFP from a green to cyan-emitting form. We report here the X-ray structures of the intact NowGFP at $\mathrm{pH} 9.0$ and $\mathrm{pH} 4.8$, and of its photoconverted variant, NowGFP_conv, at 1.35, 1.18, and $2.5 \AA$ resolution, respectively. The structure of NowGFP at pH 9.0 suggests the anionic state of Trp66 of the chromophore to be the primary cause of its green fluorescence. At both examined $\mathrm{pH}$, Trp66 predominantly adopts cis conformation; only $\sim 20 \%$ of trans conformation was observed at $\mathrm{pH} 4.8$. It was shown that Lys61 is a key residue, playing a central role in the chromophore ionization; it adopts two distinct $\mathrm{pH}$-dependent conformations. At high $\mathrm{pH}$, the side chain of Lys61 forms two H-bonds, one with the indole nitrogen of Trp66 and the other with the carboxyl group of the catalytic Glu222, enabling an indirect non-covalent connection between them that promotes Trp66 deprotonation. At low $\mathrm{pH}$, the side chain of Lys61 is directed away from Trp66 and forms an H-bond with Gln207. Here we observe that photoconversion of NowGFP is accompanied by decomposition of the Lys61 side chain. The residues Lys61, Glu222, Thr203, and Ser205 form a local H-bond network connected with the indole ring of the chromophore Trp66; replacement of any of these residues dramatically affects the spectral characteristics of NowGFP.

Keywords: fluorescent proteins, photoconversion 